\title{
Design of Ota-C Filter for Biomedical Applications
}

\author{
CH. D. Mounika 1, CH. Susmitha 2 \\ 1.(ECE,K L UNIVERSITY, INDIA),2.(EEE,RVR JC COLLEGE,INDIA)
}

\begin{abstract}
This paper presents design of operational transconductance amplifier is to amplify the ECG signal having low frequency of $300 \mathrm{~Hz}$, with the supply voltage of $0.8 \mathrm{v}$. To reduce the power dissipation of $779 n W$, by using fifth order low pass filter. The OTA-C filter is to eliminate noise voltage and increases the reliability of the system. A chip is fabricated in a $0.18 \mu \mathrm{m}$ CMOS process is simulated and measured to validate the system performance using HSPICE.
\end{abstract}

\section{Introduction}

Medical diagnostic instruments can be made into portable devices for the purpose of home care, such as the diagnosis of heart disease. These assisting devices are not only used to monitor patients but are also beneficial as handy and convenient medical instruments. Hence, for reasons of both portability and durability, designers should reduce the power consumption of assistant devices as much as possible to extend their battery lifetime. An electrocardiogram (ECG) is a test that records the electrical activity of the heart. The ECG device detects and amplifies the tiny electrical changes on the skin that are caused when the heart muscle depolarizes during each heartbeat.

Amplifier is a device for increasing the power of a signal by use of an external energy source. In an electronic amplifier, the input signal is usually a voltage or a current. A preamplifier (preamp) is an electronic amplifier that prepares a small electrical signal for further amplification or processing. A preamplifier is often placed close to the sensor to reduce the effects of noise and interference. It is used to boost the signal strength to drive the cable to the main instrument without significantly degrading the signal-to-noise ratio (SNR). When the gain of the preamplifier is high, the SNR of the final signal is determined by the SNR of the input signal.

Amplification is an essential function in most analog (and usually digital) circuits. We amplify an analog or digital signal because it may be too small to drive a load, overcome the noise of a subsequent stage, or provide logical levels to a digital circuit.

Differential operation has become the dominant choice in today's high performance analog and mixed signal circuits. A differential signal is defined as one that is measured between two nodes that have equal and opposite signal. An important advantage of differential operation over single ended signaling is higher immunity to "environmental" noise.

For the differential pair, we have $\mathrm{v}_{\text {out } 1}=\mathrm{V}_{\mathrm{DD}}-\mathrm{R}_{\mathrm{D} 1} \mathrm{I}_{\mathrm{D} 1}$ and $\mathrm{v}_{\text {out } 2}=\mathrm{V}_{\mathrm{DD}}-\mathrm{R}_{\mathrm{D} 2} \mathrm{I}_{\mathrm{D} 2}$, i.e., $\mathrm{v}_{\text {out } 1}-\mathrm{v}_{\text {out } 2}=$ $R_{D}\left(I_{D 2}-I_{D 1}\right)$. Thus, we simply calculate $I_{D 1} \& I_{D 2}$ in terms of $v_{i n 1} \& v_{i n 2}$, assuming the circuit is symmetric as show in fig $1 . \mathrm{M}_{1} \& \mathrm{M}_{2}$ are saturated, and $\lambda=0$, since the voltage at node $\mathrm{p}$ is equal to $\mathrm{v}_{\mathrm{in} 1}-\mathrm{v}_{\mathrm{GS} 1}$ and $\mathrm{v}_{\mathrm{in} 2}-\mathrm{V}_{\mathrm{GS} 2}$

For a square law device, we have

$$
\mathrm{v}_{\mathrm{in} 1}-\mathrm{v}_{\mathrm{in} 2}=\mathrm{v}_{\mathrm{GS} 1}-\mathrm{v}_{\mathrm{GS} 2}
$$

$$
\left(\mathrm{v}_{\mathrm{Gs}}-\mathrm{v}_{\mathrm{TH}}\right)^{2}=\frac{\mathrm{I}_{\mathrm{D}}}{\frac{1}{2} \mu_{\mathrm{n}} \mathrm{c}_{\mathrm{ox}} \frac{\mathrm{W}}{\mathrm{L}}}
$$

$$
\mathrm{V}_{\mathrm{GS}}=\sqrt{\frac{2 \mathrm{I}_{\mathrm{D}}}{\mu_{\mathrm{n}} \mathrm{c}_{\mathrm{ox}} \frac{\mathrm{W}}{\mathrm{L}}}}+\mathrm{v}_{\mathrm{TH}}
$$

It is instructive to calculate the slope of the characteristic, i.e., the equivalent $\mathrm{G}_{\mathrm{m}}$ of M1 \& M2 . Denoting $\mathrm{I}_{\mathrm{D} 1}-\mathrm{I}_{\mathrm{D} 2}$ and $\mathrm{v}_{\mathrm{in} 1}-\mathrm{v}_{\mathrm{in} 2}$ by $\Delta \mathrm{I}_{\mathrm{D}}$ and $\Delta \mathrm{V}_{\text {in }}$, respectively 


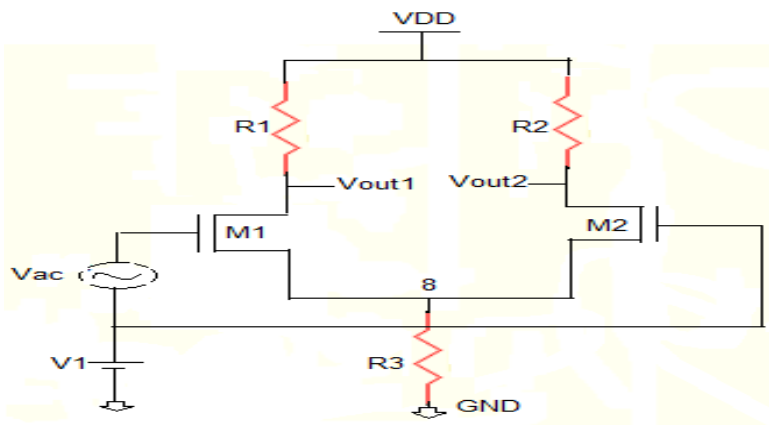

Fig. 1 Differential Amplifier with source degeneration

The small signal differential voltage gain of the circuit in the equilibrium condition as

$$
A_{v}=\sqrt{\mu_{n} c_{o x} \frac{W}{L} I_{S S} R_{D}}
$$

Where $\mu_{n}=$ mobility of electrons; $c_{o x}=$ oxide capacitance

Source degeneration- In some applications, the square law dependence of the drain current upon the gate overdrive voltage Introduces excessive non-linearity, $R_{S}$ "smoothest" this effect since it takes a portion of the gate overdrive voltage. At the limit, for $R_{S} \gg \frac{1}{g_{m}}$, the small signal does not depend on $g_{m}$ (and therefore on $I_{D S}$ ) anymore.

It is interesting to note that the approximated small signal gain (which can be easily calculated with the small signal equivalent circuit) can also be calculated as if $R_{S}$ and $\frac{1}{g_{m}}$ were two resistors in series.

Small signal gain is

$$
\mathrm{G}=-\frac{\mathrm{g}_{\mathrm{m}}}{1+\mathrm{g}_{\mathrm{m}} \mathrm{R}_{\mathrm{S}}} \mathrm{R}_{\mathrm{D}}
$$

We can see the circuit as two common source stages with degenerated resistor, and superimpose the effects. Or, even better, we can realize that the point $\mathrm{P}$ is (ideally) Ac grounded

$$
\begin{aligned}
v_{\text {out } 1} & =-g_{m} R_{D} v_{\text {in } 1} \\
v_{\text {out } 2} & =-g_{m} R_{D} v_{\text {in } 2} \\
v_{\text {out } 1-}-v_{\text {out } 2} & =-g_{m} R_{D}\left(v_{\text {in } 1}-v_{\text {in } 2}\right)
\end{aligned}
$$

We have seen that ideally in a differential pair the output voltage does not depend on the common mode input voltage. But in fact the non-infinite output impedance of the current source has an influence, since the point $\mathrm{P}$ does not behave as an $\mathrm{AC}$ ground anymore. The symmetry in this circuit suggests that we can see it as two identical half circuits in parallel.

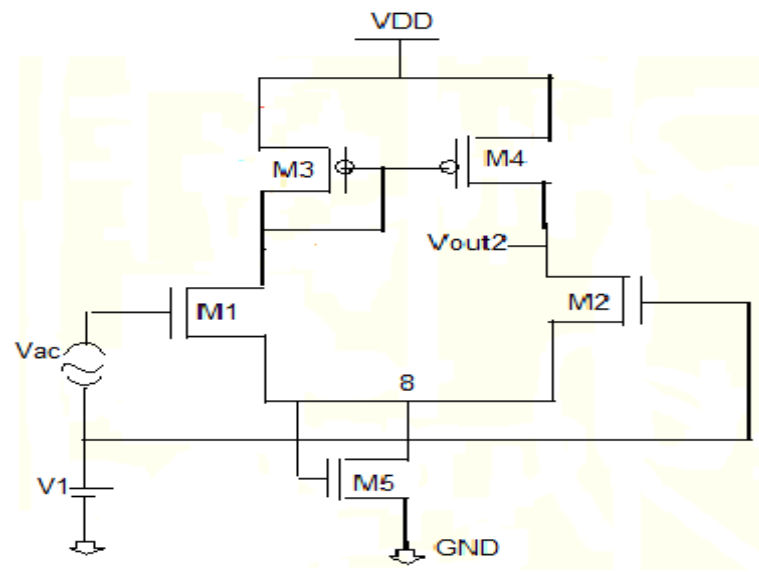

Fig. 2 Simple differential OTA

Operational Transconductance Amplifier (OTA) as shown in fig.2,is an amplifier whose differential input voltage produces an output current. Thus, it is a voltage controlled current source (VCCS). The OTA is similar to a standard operational amplifier, in that it has a high impedance, differential input stage. The term 
"operational" comes from the fact that it takes the difference of two voltages as the input for the current conversion.

The ideal transfer characteristics is

Or by taking the pre-computed difference as the input

$$
I_{\text {out }}=g_{m}\left(v_{\text {in }+}-v_{\text {in }-}\right)
$$

$$
\mathrm{I}_{\text {out }}=\mathrm{g}_{\mathrm{m}} \mathrm{v}_{\text {in }}
$$

With the ideally constant transconductanceg ${ }_{m}$ as the proportionality factor between the two. In reality the transconductance is a function of the input differential voltage and dependent on temperature. The common mode input range is also infinite, while the differential signal between these two inputs is used to control an ideal current source (i.e. the output current does not depend on the output voltage) that functions as an output. The proportionality factor between output current and input differential voltage is called "Transconductance".

A differential sinusoidal wave with a magnitude of $1 \mathrm{mVpp}$ is fed into the chip to measure the frequency response and the power spectrum with an input frequency of $50 \mathrm{~Hz}$. A low pass filter is an electronic filter that passes low frequency signals, but attenuates signals with frequencies higher than the cut-off frequency.

A fully balanced fifth-order chebyshev low pass filter, which has a $300 \mathrm{~Hz}$ cut-off frequency and $779 \mathrm{nw}$ power dissipation, is designed by the RC simulation method. The order is equal to the number of reactive components used in the network.

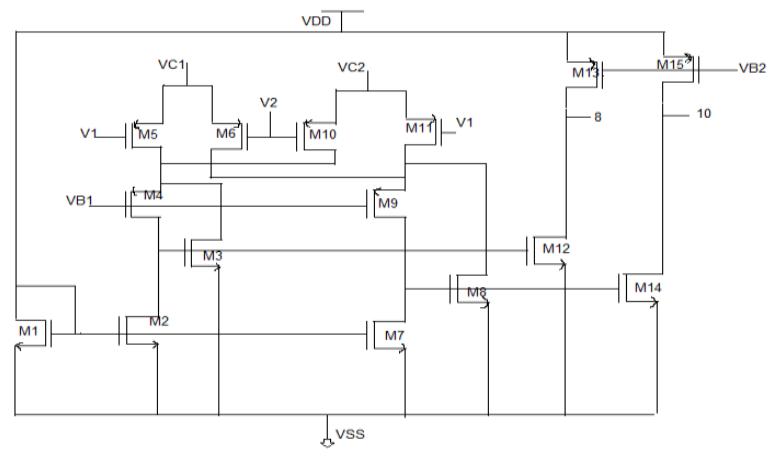

Fig.3 The Proposed OTA -C filter

Where $\mathrm{K}_{\mathrm{P}}=\mu_{\mathrm{P}} \mathrm{C}_{\mathrm{ox}} \frac{\mathrm{w}}{\mathrm{L}}$ is the transconductance parameter of the PMOS transistor, $\frac{\mathrm{w}}{\mathrm{L}}$ is the transistor aspect ratio, $\mu_{\mathrm{p}}$ is the hole mobility and $\mathrm{C}_{\mathrm{ox}}$ is the gate oxide capacitance per unit area. The differential output current is given by

$$
I_{0}=I_{01}-I_{o 2}=K_{p}\left(V_{C 2}-V_{C 1}\right)\left(V_{1}-V_{2}\right)
$$

Therefore a linear relation between the differential output current $I_{01}-I_{02}$ and the differential input voltage $\mathrm{V}_{1}-\mathrm{V}_{2}$ can be obtained $\mathrm{Vc1}$ and $\mathrm{Vc} 2$ being independent of $\mathrm{V} 1$ and $\mathrm{V} 2$. Therefore the transconductance $\mathrm{G}$ is given by

$$
\mathrm{G}=\mathrm{k}_{\mathrm{p}}\left(\mathrm{V}_{\mathrm{C} 1}-\mathrm{V}_{\mathrm{C} 2}\right)
$$

Which can be controlled by the voltage $\left(\mathrm{V}_{\mathrm{C} 2}-\mathrm{V}_{\mathrm{C} 1}\right)$. It is interesting to note that, by using the square law equation of the drain current in the saturation, the same relation between the differential output current $\mathrm{I}_{01}-\mathrm{I}_{02}$ and the differential input voltageV $V_{1}-\mathrm{V}_{2}$. The matched transistors M5, M6, M10 \& M11 are the basic transistors operating in the triode or saturation region. All other transistors are operated in the saturation region.

The role of the transistor M3 is to form a negative feedback, and vc1 \& vc2 are control voltages.

Table.4 CMOS Transistor sizing for OTA Design

\begin{tabular}{|c|l|c|}
\hline S.NO & \multicolumn{1}{|c|}{ DEVICE } & W/L $(\mu \mathrm{m})$ \\
\hline 1 & M1, M2, M5 & $1 / 0.18$ \\
\hline 2 & M3, M4 & $3 / 0.18$ \\
\hline 3 & $\begin{array}{l}\text { M1, M2, M3, } \\
\text { M7, M8, } \\
\text { M12, M14 }\end{array}$ & $6 / 0.18$ \\
\hline 4 & $\begin{array}{l}\text { M4, M5, M6, } \\
\text { M9, M10, } \\
\text { M11, M13, } \\
\text { M15 }\end{array}$ \\
\hline
\end{tabular}

For the long-term physical signal detection and monitor systems, the use of switched capacitor (SC) is a popular technique. The low sampling frequency in the kilohertz range will result in leakage, and the power 
consumption will be increased by the operational amplifiers in the SC circuits. Hence, the continuous-time operational transconductance amplifier (OTA) based filters are preferred in low-frequency applications, and the transistors inside a filter can be operated in the sub-threshold region to save power and to achieve ultra-low transconductance.

Furthermore, the fully differential structure provides a higher capability, in terms of common mode rejection and an increase of $3 \mathrm{~dB}$ in the dynamic range rather than the single end structure. In addition, all the transistors in the OTA are operated in the sub-threshold region to save the power consumption.

\section{Simulation Result:}

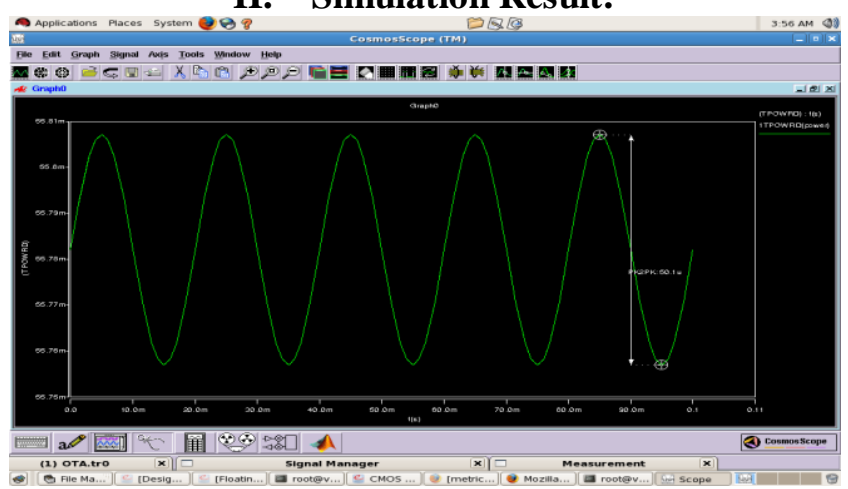

Fig.5 Hspicesimulation of Simple Differential OTA

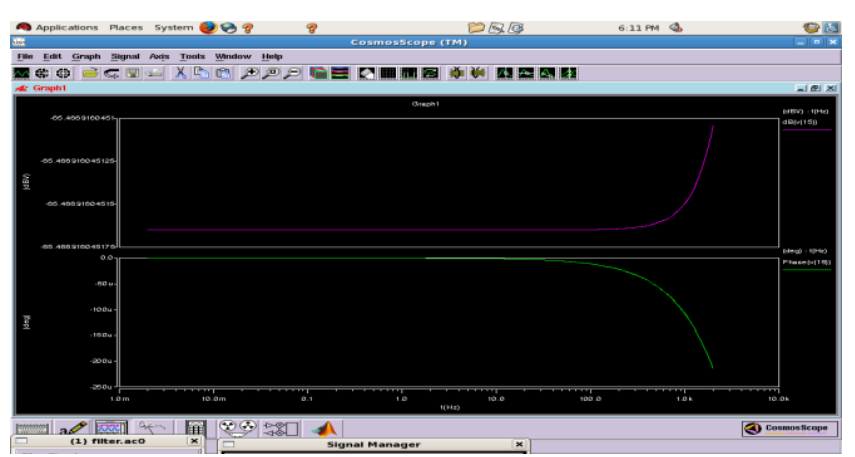

Fig.6 Frequency response with the cutoff frequency $300 \mathrm{~Hz}$ and phase

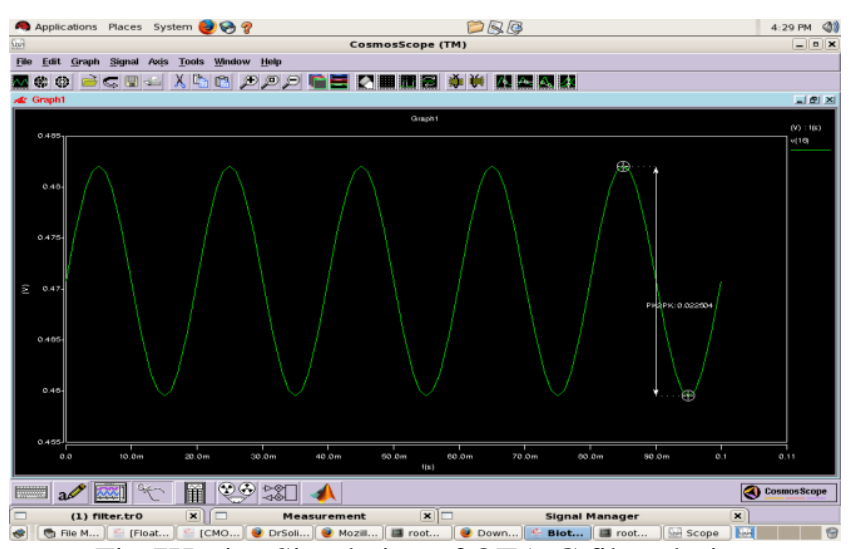

Fig.7Hspice Simulation of OTA-C filter design

Table 4.1SUMMARY OF EXPERIMENTAL RESULTS

\begin{tabular}{|c|c|c|}
\hline S.NO & EXPERIMENTAL & VALUE \\
\hline 1 & VDD & $0.8 \mathrm{~V}$ \\
\hline 2 & Technology & $180 \mathrm{~nm}$ \\
\hline 3 & Gain & $22.5 \mathrm{~dB}$ \\
\hline 4 & Input AC Supply & $1 \mathrm{mVpp}, 300 \mathrm{~Hz}$ \\
\hline 5 & Vth & $0.36 \mathrm{v}$ \\
\hline 6 & Power dissipation & $779 \mathrm{nw}$ \\
\hline 7 & CMRR & $93 \mathrm{~dB}$ \\
\hline
\end{tabular}




\section{Conclusion}

In this paper, we present "Design of OTA-C Filter for Biomedical Applications". This method of building block in SYNOPSYS Hspice tool. By the implementation of a low pass filter with a generic 0.18um CMOS technology, some significant issues of the intrinsic properties of a real OTA, such as noise reduction and finite gain. The proposed block and their application have been confirmed using Hspice simulation.

\section{References}

[1] Razavi, Behzad "Design of analog CMOS Integrated Circuits."

[2] R.Jacob Baker, Harry W.Li and David E.Boyce, "CMOS Circuit Design, Layout andSimulation."

[3] Mohamed O. Shaker, Sollman A. Mahmoud, Ahmed A. Sollman, "A CMOS Fifth-Order Low -Pass Current- Mode Filter Using a Linear Transconductor."

[4] KimmoLasanen, "Integrated analogue cmos circuits and structures for heart rate detectors and others low-voltage, low power applications."

[5] S. Koziel and S. Szczepanski, "Designof highly linear tunable CMOS-OTA for continuous-time filters."

[6] “Star-HSPICE User's Manual,” Avanti! Corp..Fremont. CA. Jun.2002, Release 2002.2.

[7] K.R. Laker and W.M.C. Sansen, Design of Analog Integrated Circuits and Systems. New York: McGraw-Hill. 1994 